\title{
Co-creating research integrity education guidelines for research institutions
}

Krishma Labib ${ }^{1}$, Natalie Evans ${ }^{1}$, Daniel Pizzolato ${ }^{2}$, Noémie Aubert Bonn ${ }^{1}$, Guy

Widdershoven ${ }^{1}$, Lex Bouter ${ }^{3,4}$, Teodora Konach ${ }^{5}$, Miranda Langendam ${ }^{6}$, Kris Dierickx ${ }^{2}$, Joeri

Tijdink $^{1,3}$

${ }^{1}$ Amsterdam University Medical Centers, Vrije Universiteit Amsterdam, Department of Ethics, Law and Humanities, Amsterdam Public Health Institute, De Boelelaan 1117, 1081HV, Amsterdam, the Netherlands

${ }^{2}$ Centre for Biomedical Ethics and Law, Department of Public Health and Primary Care, University of Leuven, Kapucijnenvoer 35, Box 7001, 3000, Leuven, Belgium2

${ }^{3}$ Vrije Universiteit Amsterdam, Department of Philosophy, De Boelelaan 1105, 1081 HV Amsterdam, the Netherlands

${ }^{4}$ Amsterdam University Medical Centers, Vrije Universiteit Amsterdam, Department of Biostatistics and Data Science, Amsterdam Public Health Institute, De Boelelaan 1117, 1081HV, Amsterdam, the Netherlands

${ }^{5}$ Austrian Agency for Research Integrity, Landstraßer Hauptstraße 9, TOP 21, 1030 Vienna, Austria

${ }^{6}$ Amsterdam University Medical Centers, University of Amsterdam, Department of Biostatistics and Data Science, Amsterdam Public Health Institute, Meibergdreef 9, 1105 AZ Amsterdam

Correspondence to k.labib@amsterdamumc.nl (+31-20444 8218 / +3120444 8266), ORCID: https://orcid.org/0000-0001-5305-8984 


\section{$\underline{\text { Abstract }}$}

Background: To foster research integrity (RI), research institutions should develop a continuous approach to RI education, addressing various target groups. To support research institutions achieve this, we developed RI education guidelines together with RI experts and research administrators, exploring similarities and differences in recommendations across target groups, as well as recommendations about RI education using approaches other than formal RI training.

Methods: We used an iterative co-creative process. After exploring the literature, we conducted four half-day online co-creation workshops with 16 participants. In the first two workshops, participants generated ideas for guidelines' content focusing on different target groups and various approaches to RI education, based on which we developed first drafts of the guidelines. Participants in the third and fourth workshop refined those drafts. We next organized a working group which further prioritized, reorganized, and optimized the content of the guidelines.

Results: We developed four guidelines on RI education focusing on a) bachelor, master and PhD students; b) post-doctorate and senior researchers; c) other RI stakeholders; as well as d) continuous RI education. Across guidelines, we recommend mandatory RI training; followup refresher training; informal discussions about RI; appropriate rewards and incentives for active participation in RI education; and evaluation of RI educational events.

Conclusions: Our work provides experience-based co-created guidance to research institutions on what to consider when developing a successful RI education strategy. Each guideline will be offered as a distinct, publicly available tool in our toolbox (https://sops4ri.eu/toolbox/) which institutions can access, adapt and implement to meet their institution-specific RI education needs.

Trial registration: https://osf.io/zej5b

Keywords: education, training, research integrity, responsible conduct of research, guidelines, institutions 


\section{Background}

Research integrity (RI) can be defined as doing research according to high professional, methodological and ethical standards (1), and is crucial for producing trustworthy research findings. Fostering RI is the joint responsibility of multiple stakeholders (2) because RI is influenced by various individual, institutional, and systemic factors, such as researchers' personal character traits (3), the departmental research culture (4,5), and assessment criteria for funding, hiring and promotion (6,7). Since researchers and their behaviors are highly dependent on the infrastructures, procedures, support systems, and research environments present at their institution, research institutions, in particular, play an important role in fostering RI (8).

One of the core responsibilities of research institutions is to provide RI education and training $(8,9)$. RI education can increase awareness about responsible research practices (RRPs) and questionable research practices (QRPs) (10). The latter category consists of practices that do not count as outright misconduct but can hamper the quality of research, e.g. selective reporting, hypothesizing after results are known, p-hacking, or poor supervision. The terms 'education' and 'training' are often used interchangeably and there are numerous ways to differentiate between them (11). In this paper, we use the term 'RI education' to refer to all approaches used to develop researchers' cognitive and moral understanding of, and skills related to, RI. Whereas we say 'training' when addressing specific formal instructional events used for RI education (e.g. courses, workshops, etc.). Thus, we see RI training as an important aspect of RI education. There is an increasing provision of RI trainings globally $(8,12-14)$, but these are typically developed without being part of a general overarching institutional RI education strategy $(14,15)$, and as such there is a risk that trainings are experienced as one-off events which have little impact on participants' long-term behavior (16). Furthermore, most existing RI educational events target $\mathrm{PhD}$ students, even though research shows that diverse stakeholders also see the need for targeting other students (i.e. at the bachelor and master level), researchers across ranks, as well as other institutional stakeholders involved in research such as RI policy makers (17). Because of the diverse needs of various research stakeholders (17), it might be that different educational strategies are required for different stakeholders (e.g. students as opposed to senior researchers). Moreover, it might be that RI training is not sufficient in providing adequate RI education, considering that RI education also takes place in informal ways, such as through supervision and socialization in the research process (17). 
It would be valuable and efficient for research institutions to develop an RI education strategy which includes various educational approaches and adequately addresses all relevant target groups (including students, researchers and other institutional stakeholders) to allow for continuous RI education (16,17). RI education guidelines, entailing recommendations and best practices, can provide considerations for institutions on what to include in their institutional RI education strategy. By 'guidelines', we refer to documents containing guidance, and by 'recommendations' we refer to the specific items in the guidelines. To ensure that guidelines are sensitive to stakeholders' actual RI education needs, they should be focused on practice and incorporate the perspectives and experiences of various research stakeholders. A co-creative approach to developing the guidelines, where stakeholders are not only consulted but also directly involved in the guideline development process, is helpful to achieve this (18).

Together with various research stakeholders, we co-created guidelines on RI education for research institutions. In this paper, we describe the development of these guidelines and reflect on them by focusing on three questions: 1) Which recommendations are applicable across various RI education target groups?; 2) Are there any specific recommendations that are applicable to some target groups but not others?; and 3) What additional recommendations are needed to increase awareness about RI in the institution, other than RI training?

\section{Methods}

The guidelines presented in this paper are the result of a combination of iterative steps used to co-create guidelines on a number of RI topics (Figure 1). Here, we focus on the methods and results relating specifically to the guidelines on the topic of RI education. To obtain a first overview of potential considerations to include in these guidelines, we used insights from several previous empirical studies, which can be seen as preliminary steps in our guideline development process $(10,17,19-22)$. We next conducted four co-creation workshops together with various stakeholders to develop the guidelines, and then formed a working group to further revise and operationalize the developed guidelines. 


\section{Preliminary steps}

- Scoping reviews

- Delphi study

- Exploratory interviews

- Focus groups

\section{Co-creation workshops}

- Content creation

- Content refinement

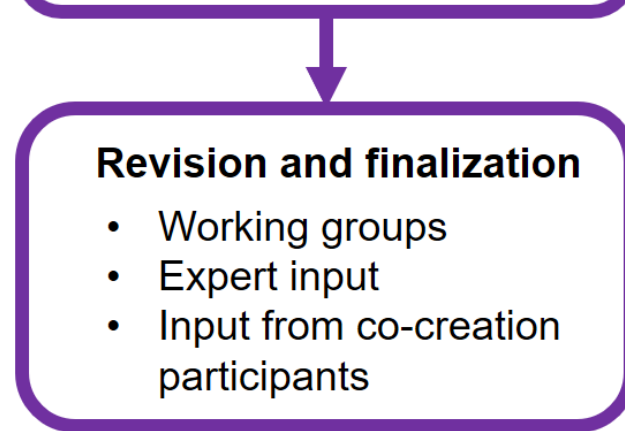

Figure 1 Guideline co-creation process

\section{Co-creation workshops}

We conducted co-creation workshops to jointly develop the RI guidelines together with various research stakeholders. The workshop methods have been described in detail elsewhere $(18,23)$. The workshops included active involvement - rather than mere consultation - of stakeholders from the onset of the guideline development process (18). We followed cocreation workshop approach as elaborated on by Sanders and Stappers (24), where stakeholders are engaged in creative workshops to jointly develop user-centered outputs. The workshops stimulated stakeholders to reflect on their experiences with RI education through the use of various interactive exercises making use of visual and textual stimuli to create ideas for guidelines, and then discuss these with others to build on each other's ideas, prioritize ideas, and make joint conclusions (18). The methods were aimed at incorporating the actual needs and perspectives of stakeholders (24), and can be considered valuable in bringing forth a broad range of ideas (18).

\section{Workshop set-up and organization}


The workshops were approved by the institutional review board of KU Leuven under dossier number G-2020011945. Prior to taking part in the workshops, participants received an information leaflet and signed an informed consent form. A detailed workshop protocol can be found on the Open Science Framework (https://osf.io/8upmb/). We conducted four cocreation workshops to develop the RI education guidelines: workshops one and two were dedicated to content creation and workshops three and four were dedicated to content refinement (Figure 2, with more details available in Appendix I). During each workshop, we asked experts to create the content of RI education guidelines separately for students, researchers, and other stakeholders to ensure that relevant differences between target groups could be addressed. Furthermore, each workshop had a section dedicated to the role of providing directed advice for RI as a form of teaching about RI, to address forms of RI education that do not fall under the category of formal RI training.

We created 'inspirations' - images or short pieces of text representing different recommendations - based on a compilation of existing ideas and recommendations about RI education from the preliminary steps (25). We sent the resulting 'inspirations' to all participants. A week later, we conducted the first two workshops aimed at content creation. During these content creation workshops, we focused on generating a broad range of ideas for the content of the guidelines. Based on the discussions in the content creation workshops, we drafted a first version of the guidelines which we sent to participants of the content refinement workshops (i.e., workshops three and four). During the content refinement workshops, we asked participants to provide general comments, additions and concerns about the guidelines, for instance regarding redundancies, gaps, unclarities, conflicting statements, etc. Following the content refinement workshops, we revised the guidelines further and sent them to participants to provide any additional comments or suggestions.

Each workshop lasted approximately 3 hours. Due to the COVID-19 pandemic, we conducted all workshops online using the virtual collaborative whiteboard software program MIRO (https://miro.com), and the video conference program Zoom (https://zoom.us/). The workshops were led by a facilitator (JT), as well as one or two co-facilitators (DP, BT, NS), and they were audio and video recorded, and transcribed. The detailed program for each workshop can be found in Appendix II. 


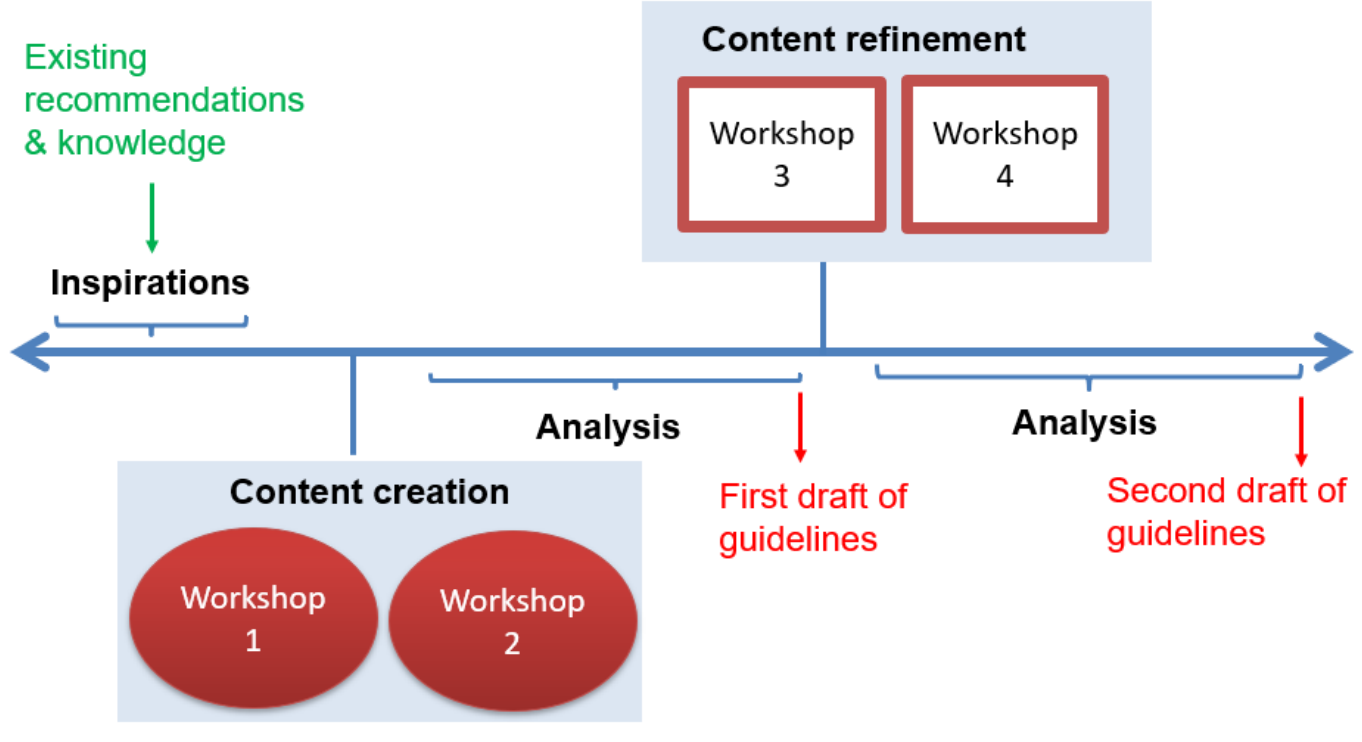

Figure 2 Guideline co-creation project process

\section{$\underline{\text { Participants }}$}

We used a purposive recruitment strategy to identify and invite participants. Participants included RI experts and research administrators representing different countries, professional roles, genders, and disciplinary fields. We aimed to recruit 4 participants per workshop to allow for in-depth discussions (18). To invite participants, we simultaneously 1) approached contacts from our networks via email, followed by snowballing, and 2) approached people listed in internal databases of RI experts (e.g. ENERI, https://eneri.eu/; EARMA, https://www.earma.org/). We recruited 16 participants in total trough this strategy (Table 1).

Table 1: Characteristics of participants

\begin{tabular}{|l|l|}
\hline Characteristics & Number of participants \\
\hline & \\
\hline Participating in each workshop & \\
\hline Workshop 1 & 4 \\
\hline Workshop 2 & 5 \\
\hline Workshop 3 & $4^{*}$ \\
\hline Workshop 4 & $5^{*}$ \\
\hline Total & 16 \\
\hline & \\
\hline Gender & \\
\hline Female & 10 \\
\hline Male & 6 \\
\hline & \\
\hline Stakeholder type & \\
\hline
\end{tabular}




\begin{tabular}{|l|l|}
\hline Research manager & 6 \\
\hline Senior researcher & 2 \\
\hline Research head & 1 \\
\hline RI coordinator & 6 \\
\hline Publisher & 1 \\
\hline & \\
\hline Country & \\
\hline Belgium & 2 \\
\hline Finland & 1 \\
\hline Germany & 1 \\
\hline Ireland & 2 \\
\hline Italy & 1 \\
\hline Lithuania & 1 \\
\hline Netherlands & 2 \\
\hline Spain & 3 \\
\hline Sweden & 1 \\
\hline Switzerland & 1 \\
\hline UK & 1 \\
\hline Total number of countries & 11 \\
\hline
\end{tabular}

*One of these participants had also contributed to the first or second workshop

\section{$\underline{\text { Analysis }}$}

$\mathrm{KL}$, IL, and NAB used inductive (26) and deductive thematic analysis (27) to analyze the results of the 'content generation' and 'content refinement workshops', respectively, through an analysis-on-the-wall approach using MIRO as described by Sanders and Stappers (24). A detailed code book including the theme and subtheme labels, and detailed descriptions and illustrative quotes for each was collaboratively developed by KL and NE

(https://osf.io/y $3 \mathrm{c} 5 \mathrm{n} /$ ). Using the insights from the analysis, we developed the first and second draft of the RI education guidelines. A detailed description of these analysis steps can be found in Appendix I.

\section{Revision and finalization of the guidelines}

After the co-creation workshops, the precise formulations of the recommendations in the guidelines needed further revision in order to be clear and usable (18). We organized a revision working group, composed of TK, GW and KL, which prioritized, reorganized, and optimized the draft recommendations in the guidelines (please see https://osf.io/f9ghj/ for more details). The working group aimed to increase the understandability, implementability, methodological soundness, and comprehensiveness of the guidelines. While revising the 
guidelines in the working group, we scrutinized the similarities and differences in the recommendations across the RI educational target groups, to ensure that the recommendations for each target group were relevant and appropriate. We also discussed the recommendations regarding increasing RI awareness through means other than formal RI training, to ensure that our RI education guidelines would capture all forms of RI education at the institution. Following this, we sent the guidelines to three external stakeholders with expertise in RI, for feedback on how to increase the implementability of the guidelines. To ensure that the feedback from participants of the co-creation project was well considered and understood in the revision process, we also sent the revised guidelines to the co-creation workshop participants as a final member check (28). Most participants responded with additional suggestions or approval of the guidelines.

\section{Results}

To adequately address the needs of students, researchers and research support staff, as well as incorporate various formal and informal approaches to RI education, we co-created four guidelines on RI education. Each guideline focused on a specific topic that we decided on together with various stakeholders $(10,21,22)$, and then finalized in the further guideline development process. The guidelines focus on: a) RI education of bachelor, master and $\mathrm{PhD}$ students (https://osf.io/z7m3v/); b) RI education of post-doctorate and senior researchers (https://osf.io/6d9ta/); c) RI education of other institutional RI stakeholders (e.g. ombudspersons, research integrity officers, etc., https://osf.io/ya3qj/); and d) continuous RI education (https://osf.io/ambg3/). Guidelines a-c focus on the steps institutions can take to provide successful education to various target groups, while guideline $\mathrm{d}$ focuses on approaches for RI education other than formal RI training. The main recommendations from each guideline are shown in Table 2, whereas the full versions of the guidelines are available in Appendices IV-VII. As can be seen, there were commonalities in the guidelines across target groups, but there were also some important points of distinction that merit discussion. 
Table 2: Key recommendations from the resulting guidelines on RI education

Guideline title

Recommendations

\section{a. Guidelines for research institutions on the RI education of bachelor, master, and PhD students}

1. Integrate RI education into the bachelor and master curriculum, making it mandatory

2. Deliver a mandatory RI course at the start of the $\mathrm{PhD}$ trajectory

3. Provide PhD students with follow-up elective courses on RI

4. Organize possibilities to discuss RI informally

5. Provide train-the-trainer education and basic qualifications for RI trainers

6. Use diverse learning environments, combining online and in-person elements in RI education

7. Focus on students' actual experiences with research rather than merely addressing theory in RI education

8. Motivate and reward students to actively take part in RI education

9. Evaluate educational programs

\section{Guideline title} Recommendations

\section{b. Guidelines for research institutions on the RI education of post-doctorate and senior researchers}

1. Deliver mandatory training about RI for researchers starting new positions

2. Provide researchers with follow-up specialized training on RI

3. Involve senior researchers in the RI training of students and junior researchers

4. Organize possibilities to discuss RI informally

5. Provide train-the-trainer education and basic qualifications for RI trainers

6. Use diverse learning environments, combining online and in-person elements in RI education

7. Consult with researchers about their RI education needs and tailor education accordingly 
8. Motivate and reward researchers to actively take part in RI education

9. Evaluate educational programs

Guideline title

Recommendations

\section{c. Guidelines for research institutions on the RI education of institutional RI stakeholders}

1. Provide institutional RI stakeholders not performing research with basic RI training

2. Organize events where RI stakeholders come together to ask questions, exchange experiences and discuss how to work together on RI

3. Provide train-the-trainer education and basic qualifications for RI trainers

4. Organize follow-up educational events when RI policies and regulations change

5. Provide opportunities for peer-to-peer learning about RI

6. Motivate and reward various RI stakeholders to actively take part in RI education

7. Evaluate educational programs

\section{Guideline title}

Recommendations

\section{d. Guidelines for research institutions on continuous RI education}

1. Provide researchers with educational RI resources to consult when needed

2. Show institutional commitment to providing continuous RI education

3. Provide researchers with contact persons who can support continuous RI education, by providing low-threshold, disciplinary-specific advice on day-to-day RI questions

4. Develop policies to foster responsible supervision and leadership

5. Develop policies for building a responsible research environment 


\section{Commonalities across target groups}

\section{Initial mandatory RI training}

Across target groups, the co-creation participants recommended mandatory RI training to ensure that everyone in the institution is well-informed about RI. More specifically, cocreation participants thought that RI training should be mandatory when starting a new academic degree program (e.g. a bachelor, master, or PhD trajectory) or a new research position (e.g. new professorship, new postdoctoral research contract, etc.). Furthermore, participants stressed the importance of having RI trainers undergo train-the-trainer courses to ensure that they are not only aware of RI theory, but are also equipped with the necessary didactic skills and tools to train students and researchers. Participants also highlighted that other RI stakeholders such as ombudspersons and RI officers would benefit from educational activities about RI, although they did not explicitly mention formal training for this. In our revision working group, we proposed to extend the recommendation for formal basic training also to these RI stakeholders when starting new positions, to ensure that they have sufficient knowledge of RI to be able to support researchers with RI.

\section{Follow-up RI training}

The co-creation workshop participants recommended that all target groups should be provided with periodic follow-up RI training. At the bachelor and master level, they suggested that discussing RI in depth during the thesis research phase would be most appropriate. For $\mathrm{PhD}$ students, it was thought that follow-up courses on discipline-specific RI topics such as data management would be most helpful in supporting students' research practice. Similarly, the co-creation workshop participants suggested that repeating follow-up disciplinary-specific training every 2-3 years to address specific RI issues such as new developments in research, would also be valuable for post-doctorate and senior researchers to keep up with the newest regulations and policies, as well as to refresh their knowledge and skills on RI. The same reasoning applied to other RI stakeholders such as RI officers, ombudspersons, and policy staff, to suggest that institutions should provide new training for these target groups every time new policies and regulations are introduced.

\section{Informal discussions about RI}

The importance of informal RI discussions were highlighted during our co-creation workshops, where many participants thought that discussing RI experiences and problems in informal meetings together with colleagues, supervisors and supervisees, would be valuable 
for continuous RI peer-to-peer learning. However, some participants were also concerned that it might be difficult for institutions to coordinate informal meetings. Therefore, in our revision working group, we recommended that institutions should stimulate and support departments and teams to organize informal events and integrate RI questions in them (e.g. by providing institutional awards for the best RI events), rather than coordinate this process themselves. The co-creation workshop participants agreed that RI education can contribute to a more responsible research culture, while an open research culture is a prerequisite for fruitful interactions during RI education. To take this consideration into account, all the RI education guidelines recommend that institutions develop policies that foster a responsible research environment, as discussed by the co-creation workshop participants and our revision working group.

\section{Motivation, incentives and rewards}

To motivate students and researchers to actively take part in RI education, our co-creation workshop participants suggested that RI educational events should emphasize the importance of RI (e.g. for research quality) and use a positive approach to RI. A positive approach entails focusing on the real-life challenges faced in research practice rather than only teaching RI theory, telling trainees what to do, or focusing on the prevention of research misconduct. The co-creation workshop participants also stressed that institutions should provide suitable incentives and rewards to ensure students and researchers are actively engaged in RI education (e.g. free lunches, certificates, promotions). Our co-creation workshop participants additionally highlighted that it is not only researchers and students who should be rewarded for taking part in RI education and contributing towards improved RI, but also other RI stakeholders such as RI trainers and officers. Although motivation, incentives and rewards were recommended for all target groups, participants stressed that these should be tailored specifically for the target group as the same incentives and rewards might not work for everyone.

\section{Evaluation of educational events}

Evaluation was seen as crucial for the continuous improvement and update of RI education. Due to potential feasibility challenges in conducting objective outcome evaluations measuring changes in behavior, the workshop participants thought that process evaluations would also be sufficient for evaluating educational events. Therefore, they suggested to evaluate educational events using subjective data, such as how useful students perceive the event to be. They also 
suggested that simple objective data not related to 'effectiveness', such as the number of individuals registering and attending optional RI trainings, could be used for evaluation purposes.

\section{Points of distinction between target groups}

\section{Bachelor, master, and PhD students}

Our co-creation participants thought that incentivizing RI education for bachelor, master and $\mathrm{PhD}$ students is relatively easy compared to other target groups. They suggested that providing students with tangible incentives - for example digital badges or other incentives tailored to students in different stages of their educational trajectory, disciplinary backgrounds and institutions - for completing trainings would suffice in motivating students to actively engage with RI education. Furthermore, they recommended providing all students with a substantial number of contact hours focused on RI, as this would ensure sufficient familiarization with RI at the start of their education about research, and would not be difficult to mandate.

\section{Post-doctorate and senior researchers}

Our co-creation workshop participants suggested that motivating post-doctorate and senior researchers to participate in RI education is difficult since researchers in these career stages are increasingly busy and have competing priorities. They stressed that institutional RI education policies should sufficiently address this concern to ensure engagement with RI education among researchers across seniority levels. Multiple recommendations on how to do this are offered in the guidelines. These include suggestions to consider RI and RI education in promotions and career assessments. The guidelines also offer other simpler suggestions such as labelling training as a 'Masterclass' rather than a 'training' to make them sound more appealing to researchers. To reduce the burden that mandatory trainings would impose, many of the co-creation workshop participants suggested to provide this target group with small training events (e.g. 1-2 hour workshops), rather than full courses. It was also thought that a 'bottom-up' approach to RI training would be valuable to make RI training attractive and relevant for post-doctorate and senior researchers. Such an approach would involve consulting with researchers beforehand to capture the RI topics they need support and help with (i.e. conducting a training needs analysis), and tailoring RI trainings accordingly. Tailoring trainings to trainees' needs was thought to be especially relevant considering that very 
experienced senior researchers might have different training needs compared to researchers in earlier stages of their career.

\section{Other institutional RI stakeholders}

Co-creation workshop participants mentioned that peer-to-peer learning is likely most suitable for the RI education of other institutional RI stakeholders (e.g. ombudspersons, RI officers, trainers, policy makers, etc.). Participants suggested that research institutions could foster peer-to-peer learning by supporting the organization of peer consultation meetings and other informal events where various RI stakeholders can come together to share experiences about RI and learn from each other. However, participants did recommend formal RI training for RI trainers, focusing not only on RI theory but also on didactical skills. They also highlighted that European level support groups and networks for institutional RI stakeholders would be valuable to address the lack of availability of RI resources at some institutions, as well as fostering the sharing of experiences across institutions and countries. Many participants suggested that it would be helpful to share RI cases and educational materials in such a network in order to learn from each other and avoid 'reinventing the wheel'. In our revision working group, we operationalized this recommendation to directly address research institutions, by suggesting that institutions provide RI stakeholders with opportunities to engage in peer-to-peer learning (e.g. by hosting networking events, providing funds or time to employees, etc.).

\section{Additional measures to increase awareness about RI}

Our co-creation workshop participants stressed that raising awareness about RI requires more than one-off RI trainings; therefore, in our revision working group, we decided to dedicate one of the RI education guidelines to continuous RI education to highlight this concern. Stimulated by advice from one of the experts we consulted in the guideline revision process, we decided to explicitly state that our definition of 'RI education' is broad and entails all means of creating awareness about RI - rather than only constituting formal education - in the preamble of the guidelines. This guideline on continuous RI education incorporates the cocreation workshop participants' recommendations regarding institutional commitment to RI education, provision of necessary educational resources, creation of policies in the institution on building a responsible research environment, inclusion of responsible supervision and mentoring, and provision of low-threshold advice to researchers about RI through informal RI 'champions' or 'stewards' as a means of increasing awareness about RI. Regarding our 
recommendations in this guideline for building a responsible research environment and fostering responsible supervision, our continuous RI education guideline links to more detailed guidance that we are developing in separate topics on the themes of research environment and supervision (23).

\section{Discussion}

We co-created institutional guidelines together with various research stakeholders regarding the RI education of a) bachelor, master and PhD students, b) post-doctorate and senior researchers, and c) other institutional RI stakeholders, as well as d) continuous RI education. These guidelines can help research institutions develop an overarching strategy for RI education that includes various educational approaches and addresses all relevant target groups. Across target groups our guidelines indicate that institutions should organize continuous RI education using multiple formal and informal educational events (e.g. workshops, courses, informal discussions, etc.) and using target-group-appropriate incentives and rewards to actively motivate trainees to stay optimally engaged in RI practices, for instance by including participation in RI education in promotion procedures for senior researchers. Furthermore, the guidelines suggest that education should focus on the concrete needs and practical challenges that participants deal with - a finding supported by a recent systematic review (29) - , and use regular process evaluations to ensure constant updating and improvement. Moreover, our guidelines highlight that a holistic RI education approach will not only require provision of formal RI trainings, but also additional educational approaches (e.g. responsible supervision) so as to support continuous education.

Given that current RI education often consists of stand-alone courses on RI (12), the implementation of continuous RI education will require substantial effort and commitment by research institutions to organize, design and deliver additional RI training events to various target groups. Although this could be perceived as a high burden by research institutions (22), we believe this commitment is necessary given that it is highly unlikely that a single course or workshop will be sufficient in influencing trainees' perceptions of and behaviors relating to RI (14). However, further empirical research on the effects that RI education has on researchers' behavior is urgently needed to confirm this. To increase the feasibility of providing continuous RI education, institutions could make use of learning approaches utilizing different learning mediums where possible; they could consider using already existing openly accessible online RI trainings and resources, they could integrate relevant RI discussions in existing research courses, workshops, and department meetings, or they could 
cooperate with external trainers and institutions to share the provision of RI education. This recommendation is supported by evidence suggesting that blended learning approaches are highly effective for ethics instruction (30).

While it can be assumed that mandating RI training for senior researchers is likely to meet resistance $(17,31)$, our co-creation workshop participants recommend mandatory RI training for senior as well as junior researchers. This is based on the view that mandatory training is the only way to ensure that all researchers - rather than only those who already consider RI as important - take part in RI education. To reduce the potential resistance and to ensure that trainees are actively engaged in RI education, the RI education guidelines stress the importance of providing suitable incentives and rewards for participating in RI training (e.g. tying RI education to promotions, using tangible rewards, etc.). Our guidelines further suggest to tailor incentives and rewards to their target group: a finding that is in line with existing literature suggesting that effective incentives and rewards are different for junior than for senior researchers $(17,31)$. Rewarding and incentivizing participation in RI education is also in line with other existing initiatives in the research community which state that researcher evaluations should consider a broader range of contributions and should value responsible research practices $(6,32)$.

Motivation to actively participate in training will also depend on the extent to which the RI training appeals to the needs of each target group. Therefore, our guidelines stress the importance of providing RI education that focuses on the specific needs and challenges of the education target group. Focusing on real life cases of RI dilemmas that come up in research practice when teaching students about RI can help increase the relevance of RI training and has been suggested by others as well $(14,29,31)$. Our recommendation to use a training-needsanalysis to ensure that post-doctoral and senior researchers can determine what should be included in their RI trainings and how, rather than following trainings focusing on methods and context predetermined as relevant for them by trainers, has to our knowledge, not been discussed in previous literature. Especially considering that researchers of various disciplines and ranks (e.g. full professors as compared to less experienced post-doctorate researchers) may have different needs (33), using such a bottom-up, tailored approach to RI education is likely to be valuable, albeit the associated financial and time costs present challenges for implementation.

To ensure that RI education is continuously updated and improved over time, the RI education guidelines emphasize the importance of evaluating RI educational events. However, our results also suggest that evaluating educational events on 'effectiveness' will likely be 
difficult, if not impossible, indicating the need for institutions to prioritize subjective processrelated evaluations (e.g. on perceptions of training usefulness) over outcome-oriented evaluations (e.g. relating to changes in actual behaviors). This approach to evaluation might seem dissatisfactory for trainers who would like to develop RI trainings based on solid empirical outcome research, as well as for institutions who would like to know that their RI education policies are actually impacting research practice. However, we would argue that it is not the responsibility of single research institutions to provide full insight into what makes RI education effective; a focus on subjective evaluations is more feasible and can still provide valuable information to trainers.

Evaluations on subjective data like stakeholder experiences can provide valuable information about the contextual mechanisms and processes that influence the success of educational initiatives (34). Stakeholder experiences are furthermore relevant for identifying outcome parameters, and for deepening understanding of outcomes (e.g. regarding whether outcomes are recognizable or explainable). Taking this into account, ideally a combination of subjective and outcome evaluations provide the most insights about the value of RI educational events. There are substantial current efforts to find strategies to measure RI training effectiveness on outcomes such as improvements in moral reasoning and changes in behavior (e.g. Abdi, Fieuws, et al. 2021; Katsarov et al. 2020; Watts et al. 2017); these can provide institutions with information on the effectiveness of RI education and supplement institutional efforts in evaluating various educational programs. Once the effects on researcher behavior of a specific approach to RI education are established there is no need to repeat that research in every institution.

\section{Strengths and limitations}

The guidelines we discuss in this paper provide an overview of what to include in research institutions' overarching RI education strategy. The guidelines are a result of an iterative cocreative research process, involving various potential lead-users from different parts of Europe. As such, the guidelines have been developed with a focus on incorporating various research stakeholders' actual RI education needs and perspectives. Although we included insights gained from consultations with various stakeholders including junior and senior researchers into the guidelines based on previous studies $(17,21)$, the active co-creators of the project represent the guideline lead-users (e.g. RI officers and trainers) rather than end-users (e.g. junior and senior researchers). Actual testing of the guidelines in a number of research institutions will be necessary to further refine the guidelines and make them implementable on 
a large scale. Institutions interested in using the guidelines will need to take into account costs, local capacity, cultural issues, and context-specific factors during implementation of the guidelines. Implementation of the recommendations will likely vary between institutions which already provide some RI educational programs and those that do not.

\section{Conclusions}

Our work provides experience-based co-created guidance to research institutions on important considerations for developing a successful RI education strategy. Our guidelines on RI education address the needs of students, researchers and other RI stakeholders, and take into account various approaches to RI education. In the guidelines, we recommend mandatory RI training; follow-up refresher training; informal discussions about RI; appropriate rewards and incentives for active participation in RI education; and evaluation of RI educational events across target groups. Each of our four guidelines can be considered as a distinct tool that institutions can access, adapt and implement to meet their institution-specific RI education needs. Research institutions across and outside of Europe can use our guidelines as tools to strengthen their RI education efforts and consequently contribute towards better quality and more trustworthy research.

\section{List of abbreviations}

RI - Research integrity

RRPs - responsible research practices

QRPs - questionable research practices 


\section{Declarations}

\section{Ethics approval}

The workshops were approved by the institutional review board of KU Leuven under dossier number G-2020011945. Prior to taking part in the workshops, participants received an information leaflet and signed an informed consent form.

\section{Consent for publication}

Not applicable

\section{Availability of data and materials}

Due to privacy reasons, the co-creation workshop transcripts used for this article are not publicly available. However, we have made the anonymized code book, including quotes, publicly available on the Open Science Framework: https://osf.io/y3c5n/. We excluded all descriptions about the characteristics of the quote owners (e.g. country, role, gender), to prevent the identifiability of the data, considering that the majority of the co-creators are acknowledged in this paper.

\section{Competing interests}

The authors have no conflicts of interest to declare.

\section{Funding}

This study was funded by the European Commission HORIZON 2020 framework programme for Research and Innovation under Grant Agreement No 824481. The funder was not involved in the design, conduct, analysis or interpretation of the study.

\section{Authors' contributions}

KL contributed to the preliminary steps of the guideline development process, designed the co-creation workshops and analyzed the resulting data, led the working group for the refinement of the guidelines, and drafted and revised the manuscript. NE contributed towards the preliminary steps of the guideline development process, the co-creation study design and data analysis, and revision of the manuscript. DP contributed towards the co-creation study design and data collection, and revision of the manuscript. NAB contributed towards the cocreation workshop data analysis, designed the protocol used in the revision working group of 
the guidelines, and revised of the manuscript. GW, LB and KD contributed to the co-creation workshop study design, and revision of the manuscript. GW and LB were additionally involved in the guideline development process. GW, together with TK, was a member of the revision working group of the guidelines; TK also revised the manuscript. ML contributed towards the design of the protocol used in the guideline revision working group and revised the manuscript. JT contributed to the preliminary steps of the guideline development process, revised the co-creation workshop study protocol and collected the resulting data, revised the manuscript, and supervised the work.

\section{Acknowledgements}

16 co-creators participated in creating these guidelines. Among those, the following consented to be acknowledged: Sirpa Aalto, Eva Casamitjana-Martínez, Susan Hommerson, Jonas Åkerman, Joana Porcel, Peter Apers, Ruth Moran, Maruxa Martínez-Campos, Marie Soulière, Helga Nolte, Inge Lerouge, Stephanie Boughton, Leonas Balaševičius, Fiona Brennan, Francesca Tomasi.

We would like to thank Pieter Jan Stappers and Katinka Bergema for their valuable advice on co-creation methodology and their contribution towards the study design, as well as the three experts who provided us with input on the revision of the guidelines: Mariëtte van den Hoven, Julia Priess-Buchheit, and Malcolm MacLeod. We would also like to thank all members of the SOPs4RI project who contributed to the various stages of the guideline development process, and the co-creation project including Iris Lechner, Nik Claesen, Borana Taraj, and Nikolaos Skoulikaris, and Roshni Jegan. 


\section{References}

1. Boehme O, Föger N, Hiney M, Peatfiled T, Petiet F. Research Integrity Practices in Science Europe Member Organisations. 2016.

2. Bouter L. Fostering responsible research practices is a shared responsibility of multiple stakeholders. J Clin Epidemiol. 2018;96:143-6.

3. Tijdink J, Bouter L, Veldkamp CLS, van de Ven PM, Wicherts JM, Smulders YM. Personality traits are associated with research misbehavior in Dutch scientists: a crosssectional study. PLoS One. 2016;11(9):e0163251.

4. Haven T, Tijdink J, Martinson BC, Bouter L. Perceptions of research integrity climate differ between academic ranks and disciplinary fields: Results from a survey among academic researchers in Amsterdam. PLoS One. 2019;14(1):e0210599.

5. Joynson C, Leyser O. The culture of scientific research. F1000Research. 2015;4.

6. Aubert Bonn N, Bouter L. Research assessments should recognize responsible research practices - Narrative review of a lively debate and promising developments. OSF Preprints. 2021. DOI: 10.31222/osf.io/82rmj

7. Titus S, Wells JA, Rhoades LJ. Repairing research integrity. Nature. 2008;453(7198):980-2.

8. Mejlgaard N, Bouter L, Gaskell G, Kavouras P, Allum N, Bendtsen A-K, et al. Research integrity: nine ways to move from talk to walk. Nature Publishing Group; 2020.

9. All European Academies. The European Code of Conduct for Research Integrity [Internet]. All European Academies; 2017. Available from: https://www.allea.org/wpcontent/uploads/2017/05/ALLEA-European-Code-of-Conduct-for-Research-Integrity2017.pdf

10. Labib K, Roje R, Bouter L, Widdershoven G, Evans N, Marušić A, et al. Important topics for fostering research integrity by research performing and research funding organizations: a delphi consensus study. Sci Eng Ethics. 2021;27(4):1-22.

11. Masadeh M. Training, education, development and learning: what is the difference? Eur Sci J. 2012;8(10).

12. Abdi S, Pizzolato D, Nemery B, Dierickx K. Educating PhD Students in Research Integrity in Europe. Sci Eng Ethics. 2021;27(1):1-19.

13. Evans N, Marusic A, Foeger N, Lofstrom E, van Hoof M, Vrijhoef-Welten S, et al. Virtue-based ethics and integrity of research: train-the-trainer programme for upholding the principles and practices of the European Code of Conduct for Research Integrity (VIRT2UE). Res Ideas Outcomes. 2021;7:e68258.

14. Kalichman M. Rescuing responsible conduct of research (RCR) education. Account Res. 2014;21(1):68-83.

15. Kalichman M, Plemmons DK. Reported goals for responsible conduct of research courses. Acad Med. 2007;82(9):846-52.

16. Barnes BE, Friedman CP, Rosenberg JL, Russell J, Beedle A, Levine AS. Creating an infrastructure for training in the responsible conduct of research: the University of 
Pittsburgh's experience. Acad Med. 2006;81(2):119-27.

17. Labib K, Evans N, Roje R, Kavouras P, Reyes Elizondo A, Kaltenbrunner W, et al. Education and training policies for research integrity: Insights from a focus group study. Sci public policy. 2021;(scab077).

18. Labib K, Pizzolato D, Stappers PJ, Evans N, Lechner I, Widdershoven G, et al. Using co-creation for guideline development - How why and when? OSF Prepr [Internet]. 2021; Available from: https://osf.io/cg3rw/

19. Gaskell G, Ščepanović R, Buljan R, Utrobičić A, Marušić A, Reyes Elizondo A, et al. D3.2: Scoping reviews including multi-level model of research cultures and research conduct [Internet]. Marušić A, editor. 2019. Available from: https://www.sops4ri.eu/wp-content/uploads/D3.2_Scoping-reviews-including-multilevel-model-of-research-cultures-and-research-conduct.pdf

20. Ščepanović R, Labib K, Buljan I, Tijdink J, Marušić A. Practices for Research Integrity Promotion in Research Performing Organisations and Research Funding Organisations: A Scoping Review. Sci Eng Ethics. 2021;27(1):1-20.

21. Ščepanović R, Tomić V, Buljan I, Marušić A. D3. 3: Report on the results of explorative interviews [Internet]. 2019. Available from: https://www.sops4ri.eu/wpcontent/uploads/D3.3_Report-on-the-results-of-the-explorative-interviews.pdf

22. Sørensen MP, Ravn T, Marušić A, Elizondo AR, Kavouras P, Tijdink JK, et al. Strengthening research integrity: which topic areas should organisations focus on? Humanit Soc Sci Commun. 2021;8(1):1-15.

23. Pizzolato D, Dierickx K, Tijdink J, Labib K, Lechner I, Bonn NA, et al. D4.4: Report on the Co-Creation Workshops [Internet]. 2021. Available from: https://sops4ri.eu/wpcontent/uploads/D4.4_Report-on-the-Co-Creation-Workshops.pdf

24. Sanders E, Stappers PJ. Convivial toolbox: generative research for the front end of design. Amsterdam: BIS Publishers; 2012.

25. Lechner I, Tijdink J, Sørensen MP, Ravn T, Bendtsen A-K, Labib K, et al. D4.3: Second version of SOPs and guidelines [Internet]. 2020. Available from: https://sops4ri.eu/wp-content/uploads/D4.3_Second-version-of-SOPs-and-guidelines1.pdf

26. Boyatzis RE. Transforming qualitative information: Thematic analysis and code development. Thousand Oaks: SAGE Publications Inc; 1998.

27. Crabtree BF, Miller WF. A template approach to text analysis: developing and using codebooks. In: Crabtree BF, Miller WF, editors. Doing qualitative research. Newbury Park, CA: SAGE Publications Inc; 1992. p. 163-77.

28. Thomas DR. Feedback from research participants: are member checks useful in qualitative research? Qual Res Psychol. 2017;14(1):23-41.

29. Katsarov J, Andorno R, Krom A, van den Hoven M. Case discussions are not the gold standard of Research Integrity training [Internet]. 2020. Available from: https://h2020integrity.eu/case-discussions-are-not-the-gold-standard-of-researchintegrity-training/

30. Todd EM, Watts LL, Mulhearn TJ, Torrence BS, Turner MR, Connelly S, et al. A 
meta-analytic comparison of face-to-face and online delivery in ethics instruction: the case for a hybrid approach. Sci Eng Ethics. 2017;23(6):1719-54.

31. Fanelli D. MLE on Research Integrity - Training \& Education - Thematic Report No 4 [Internet]. 2019. Available from:

https://rio.jrc.ec.europa.eu/sites/default/files/report/MLE RI No4 - Training and education.pdf

32. Moher D, Bouter L, Kleinert S, Glasziou P, Sham MH, Barbour V, et al. The Hong Kong Principles for assessing researchers: Fostering research integrity. PLoS Biol. 2020;18(7):e3000737.

33. ENERI. Report of Stakeholder/Focus Group Workshop in Athens [Internet]. 2017. Available from: https://eneri.eu/wp-content/uploads/2018/06/Report-StakeholderWorkshop-Athens.pdf

34. Hamza DM, Ross S, Oandasan I. Process and outcome evaluation of a CBME intervention guided by program theory. J Eval Clin Pract. 2020;26(4):1096-104.

35. Abdi S, Fieuws S, Nemery B, Dierickx K. Do we achieve anything by teaching research integrity to starting PhD students? Humanit Soc Sci Commun. 2021;8(1):1-7.

36. Watts LL, Medeiros KE, Mulhearn TJ, Steele LM, Connelly S, Mumford MD. Are ethics training programs improving? A meta-analytic review of past and present ethics instruction in the sciences. Ethics Behav. 2017;27(5):351-84. 